\title{
Role of substorm-associated impulsive electric fields in the ring current development during storms
}

\author{
N. Yu. Ganushkina ${ }^{1}$, T. I. Pulkkinen ${ }^{1}$, and T. Fritz ${ }^{2}$ \\ ${ }^{1}$ Finnish Meteorological Institute, Geophysical Research Division, P.O. Box 503, FIN-00101 Helsinki, Finland \\ ${ }^{2}$ Boston University, Department of Astronomy, 725 Commonwealth Ave., Boston, MA 02215, USA
}

Received: 12 May 2004 - Revised: 1 October 2004 - Accepted: 6 December 2004 - Published: 28 February 2005

\section{Abstract.}

Particles with different energies produce varying contributions to the total ring current energy density as the storm progresses. Ring current energy densities and total ring current energies were obtained using particle data from the Polar CAMMICE/MICS instrument during several storms observed during the years 1996-1998. Four different energy ranges for particles are considered: total $(1-200 \mathrm{keV})$, low (1-20 keV), medium (20-80 keV) and high (80-200 keV). Evolution of contributions from particles with different energy ranges to the total energy density of the ring current during all storm phases is followed. To model this evolution we trace protons with arbitrary pitch angles numerically in the drift approximation. Tracing is performed in the large-scale and small-scale stationary and time-dependent magnetic and electric field models. Small-scale time-dependent electric field is given by a Gaussian electric field pulse with an azimuthal field component propagating inward with a velocity dependent on radial distance. We model particle inward motion and energization by a series of electric field pulses representing substorm activations during storm events. We demonstrate that such fluctuating fields in the form of localized electromagnetic pulses can effectively energize the plasma sheet particles to higher energies $(>80 \mathrm{keV})$ and transport them inward to closed drift shells. The contribution from these high energy particles dominates the total ring current energy during storm recovery phase. We analyse the model contributions from particles with different energy ranges to the total energy density of the ring current during all storm phases. By comparing these results with observations we show that the formation of the ring current is a combination of large-scale convection and pulsed inward shift and consequent energization of the ring current particles.

Key words. Magnetospheric physics (Energetic particles, trapped; Electric fields; Storms and substorms)

Correspondence to: N. Yu. Ganushkina

(nataly.ganushkina@fmi.fi)

\section{Introduction}

The $\boldsymbol{E} \times \boldsymbol{B}$ drift is responsible for the basic transport and acceleration of ions moving from the magnetotail and the plasma sheet to the inner magnetosphere. Approaching the inner magnetosphere, the gradient and curvature drifts add to the particle transport. Particles entering the inner magnetosphere from the plasma sheet are either trapped to Earthorbiting trajectories or drift to the magnetospheric boundary and subsequently leave the magnetosphere. The main portion of the Earth's ring current is carried by these trapped energetic ions in the energy range of $10-200 \mathrm{keV}$. As the net westward-flowing ring current decreases the horizontal component of the geomagnetic field at the Earth's surface, the $D_{s t}$ index constructed from ground magnetic measurements is commonly used as a measure of space storm intensity.

The sharp increase of energetic (a few tens to hundreds of $\mathrm{keV}$ ) particle flux in the near-Earth tail is one of the most important manifestations of the substorm expansion phase (Arnoldy and Chan, 1969; Baker et al., 1982; Reeves et al., 1991). Several models have been proposed to explain the rapid injection of energetic particles. McIlwain (1974) suggested an injection boundary model, where a spatial boundary forms during the injection process separating newly injected or energized plasma from the preexisting, undisturbed plasma. Moore et al. (1981) suggested a convection surge model, in which a compressional wave propagates from the tail into the inner magnetosphere regions. Birn et al. (1997), by tracing test particle orbits in the dynamic fields from a three-dimensional MHD simulation, found that most energization is caused by betatron acceleration as particles are transported into the stronger magnetic field region by a timedependent dawn-to-dusk electric field.

The temporal and spatial structure of the electric field is important in understanding how the energetic particle injections are formed and how the particles are accelerated. Observations show that substorm-associated electric fields usually display a very complicated behavior (Maynard et al., 1996). Large, transient electric fields appear in the plasma sheet during the substorm expansion phase (Aggson et al., 1983; Cattell and Mozer, 1984; Rowland and Wygant, 1998; 
Wygant et al., 1998). The enhanced electric fields are impulsive with amplitudes up to $20 \mathrm{mV} / \mathrm{m}$ (Wygant et al., 1998), and coincident with the braking of the fast flows and the magnetic field dipolarization in the central plasma sheet (Tu et al. (2000) and references therein).

The origin of strong transient electric fields at substorm onset and their relationship to the magnetic field dipolarization is still an open question. At the same time, several models have been proposed to represent the substorm-associated electromagnetic fields (Li et al., 1998; Zaharia et al., 2000; Sarris et al., 2002). In the Li et al. (1998) model the electric field was modeled as a time-dependent Gaussian pulse with a purely azimuthal electric field component. The model was built on the idea that a perturbation further out in the magnetotail propagates inward, probably in the form of bursty bulk flows, and produces dipolarization and particle injection. Sarris et al. (2002) introduced a pulse velocity decreasing with radial distance, which gave good agreement with the observed electron injections at geostationary orbit.

In the present paper we study the role of the substormassociated electric fields in the transport of the plasma sheet protons to the ring current and their energization to higher energies $(>80 \mathrm{keV})$. In the observational part, we discuss the methodology of deriving the ring current energy density from Polar CAMMICE/MICS data for different energy ranges. We follow the evolution of contributions from protons with different energy ranges to the total energy density of the ring current during different storm phases. We model the injections by tracing protons numerically in the drift approximation in several combinations of the large-scale and smallscale stationary and time-dependent magnetic and electric field models and using different initial conditions. The storm on 2-4 May 1998 is used to discuss the conditions that are necessary for the magnetic and electric fields to account for the observed earthward transport and energization of ions.

\section{Proton ring current energy density and total en- ergy calculated from Polar CAMMICE/MICS parti- cle measurements}

\subsection{Instrumentation: Polar CAMMICE/MICS}

During the years 1996-1998 the Polar spacecraft was on an $\sim 86^{\circ}$ inclination elliptical orbit with $9 R_{E}$ apogee, $1.8 R_{E}$ perigee, and 18 -h orbital period. The orbit apogee was over the northern polar region. The satellite spin axis is normal to the orbit plane, to enable the particle instruments to map the complete charged particle distribution function, including the loss cone. The Charge and Mass Magnetospheric Ion Composition Experiment on board Polar was designed to measure the charge and mass composition of particles within the Earth's magnetosphere over the energy range of $6 \mathrm{keV} / \mathrm{Q}$ to $60 \mathrm{MeV} / \mathrm{Q}$ (Wilken et al., 1992). CAMMICE consists of two sensor systems: the Magnetospheric Ion Composition Sensor and the Heavy Ion Telescope. The MICS sensor identifies each ion from time-of-flight measurements, giving the energy per charge and total energy. An electrostatic analyzer allows entry of the ions in one of 32 energy/charge steps in the range of $1-200 \mathrm{keV} / \mathrm{e}$. The particle events are analyzed on board to obtain their mass and mass/charge. The counts of the major ion species are accumulated into scalers, with a full 32-channel energy spectrum being telemetered once every $202 \mathrm{~s}$. Figure 1 shows an example of Polar CAMMICE/MICS measurements in the form of energy-time spectrograms and pitch-angle distributions for protons (upper two panels), total He (middle two panels) and total O (bottom two panels) during 4 May 1998, 19:00-24:00 UT. These data are used here to study the properties of the proton ring current population during storms.

\subsection{Ring current energy density: storm statistics}

We used pitch-angle averaged fluxes measured by Polar CAMMICE/MICS during 1996-1998. The data were tagged with Polar orbital information and $L$-shell values obtained from the Polar database. The $L$ values were calculated assuming the dipole magnetosphere, which can introduce mapping errors, especially during disturbed periods. However, as accurate mapping during storm times is still not possible, using the dipole mapping was judged to be the best solution.

The energy density computations were made similarly to those introduced by Pulkkinen et al. (2001). To accurately account for the loss cone, pitch angle corrections $j^{e q}(E, \alpha)=j^{\text {meas }}(E, \pi / 2) \sin ^{n} \alpha$, where $j^{e q}$ and $j^{\text {meas }}$ are the equatorial and measured fluxes and $\alpha$ is the pitch-angle, should be introduced. The exponent $n$ typically becomes large only for energies much higher than those covered by the CAMMICE/MICS instrument, and hence the value $n=0$ was assumed in the statistical examination with the assumption that the underestimation of the fluxes is small. The energy density per unit volume $w(L)$ of the ring current particles in the energy range of ( $\left.E_{\min }, E_{\max }\right)$ between $L=3$ and $L=8$ is computed from

$w(L)=2 \pi \sqrt{2 m q} \int_{E_{\min }}^{E_{\max }} d E \sqrt{E} j(E, L)$,

where $m$ is the particle mass, $q$ is the particle charge state, $E$ is the particle energy, $j(E, L)$ is the measured particle differential flux, and $L$ is the McIlwain $L$-parameter. The total ring current energy, $W_{R C}$, is computed by integration over the ring current volume $d V$,

$W_{R C}=\int_{V} w(L) d V$,

$d V=2 R_{E}^{3} L^{2} \sqrt{1-\frac{1}{L}}\left(\frac{1}{7 L^{3}}+\frac{6}{35 L^{2}}+\frac{8}{35 L}+\frac{16}{35}\right) d L d \phi$,

where the Earth's radius is $R_{E}=6371 \mathrm{~km}$ and $\phi$ is the local time.

The ring current energies were computed separately for the protons in four energy ranges: all energies (1-200 keV), low energies (0-20 keV), medium energies (20-80 keV) and high 


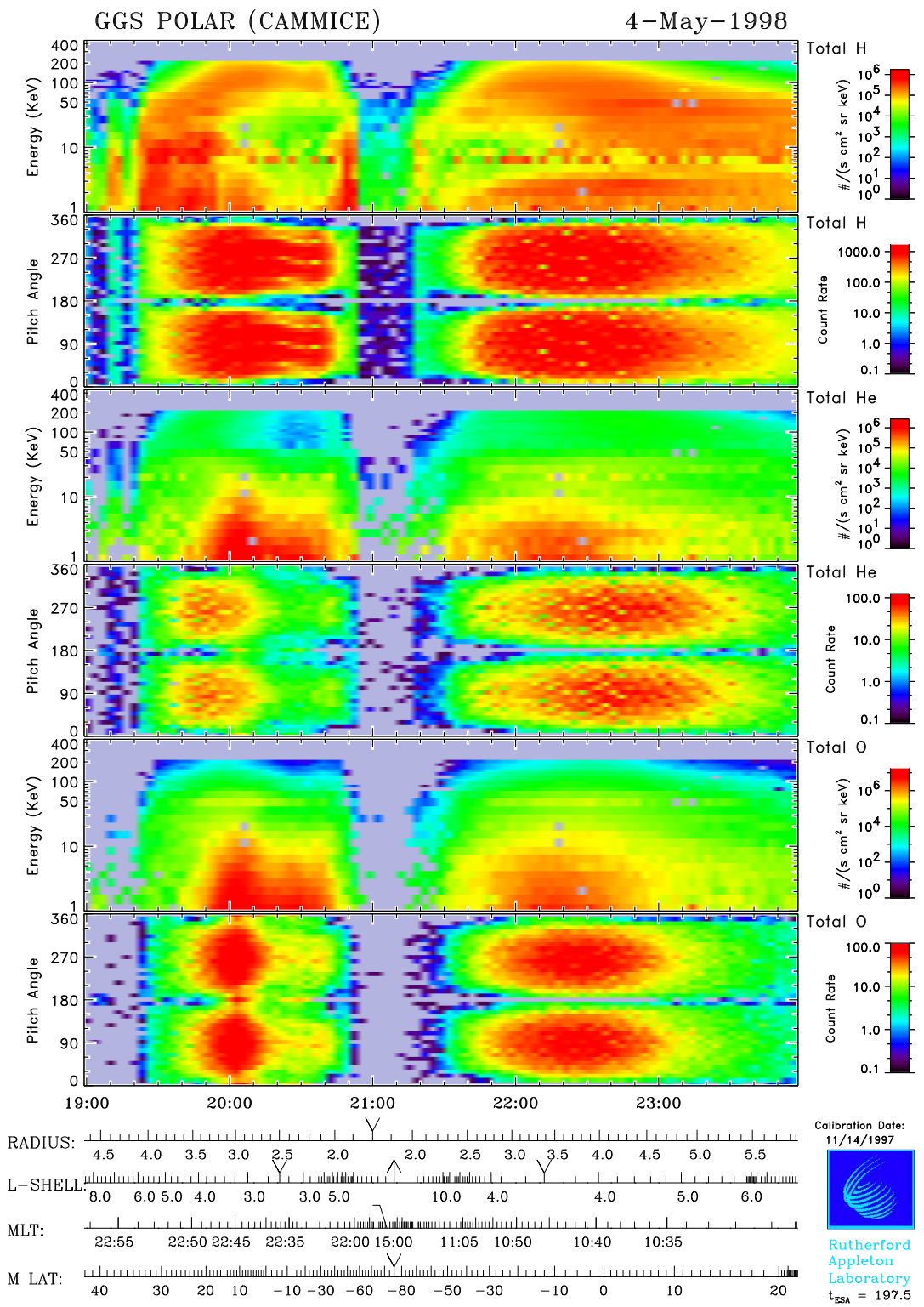

Fig. 1. Example of Polar CAMMICE/MICS measurements: Energy-time spectrograms and pitch-angle distributions for protons (upper two panels), total He (middle two panels) and total O (bottom two panels) during 4 May 1998, 19:00-24:00 UT.

energies (80-200 keV). Furthermore, each observation was categorized in three bins according to the phase of the storm: "initial phase" (positive $D_{s t}$ deflection), "main phase" (steep decrease in the $D_{s t}$ index) and "recovery phase" (gradual recovery of the $D_{s t}$ index).

Figure 2 shows statistical results for 27 storms selected during the period of 1996-1998. Contributions to the total ring current energy from low energy protons (upper panels, blue triangles), medium energy protons (middle panels, green diamonds) and high energy protons (bottom panels, red rectangles) for the (a) initial phase, (b) main phase and (c) recovery phase of the storms. Linear fits are shown by solid black lines in all panels. During the initial phase of the storm, the variance in the relative contributions is large, and the means do not differ significantly for the different energy ranges. There is a slight trend showing an increase in the contribution from medium energy protons and a decrease in the contribution from high energy protons with a decrease in the $D_{s t}$ index approaching the storm main phase. During the storm main phase, the contribution from low energy protons decreases when the storm intensifies and the $D_{s t}$ index decreases, medium energy protons show no dependence on the $D_{s t}$, and the contribution from high energy protons increases. The notable feature is that during the storm main phase the main contribution to the proton ring current energy comes from the medium energy protons (about $60 \%$ ). The high and low energy protons contribute no more than $20 \%$. On the other hand, during the recovery phase the high energy protons play a dominant role, their contributions are larger (up to $80 \%$ ), or comparable (60-30\%), to those of the medium 


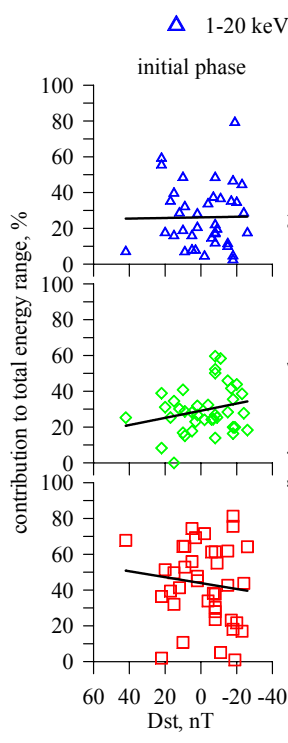

(a)

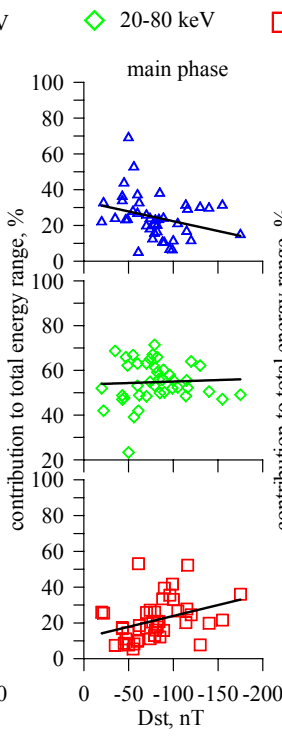

(b)

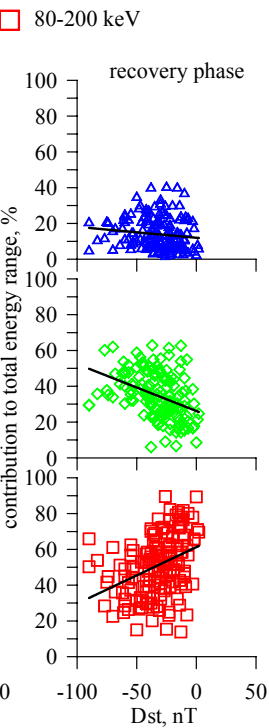

(c)
Fig. 2. $D_{s t}$-dependent contributions in percents to the ring current energy (1-200 keV) from protons with low $(0-20 \mathrm{keV}$, blue triangles, upper panels), medium (20-80 keV, green diamonds, middle panels) and high (80-200 keV, red rectangles, bottom panels) for (a) initial, (b) main and (c) recovery storm phases calculated using Polar CAMMICE/MICS measurements during 27 storms during 1996-1998. The linear fits are shown by solid black lines in all panels.

energies. When $D_{s t}$ index recovers, the contributions from low and medium energy protons decrease, whereas the contribution from high energy protons continues to increase.

\section{1-7 May 1998 storm: observations}

3.1 Solar and solar wind activity and magnetospheric response

The storm in early May, 1998, was initiated from an extended period of solar activity which started on 29 April 1998. There were several coronal mass ejections during the period: on 29 April (17:00 UT), 1 May (23:40 UT), 2 May (05:30 UT) and 4 May (02:00 UT). Figure 3 shows (a) the $B_{z}$ component of the interplanetary magnetic field, (b) $V_{x}$ component of the solar wind velocity, (c) solar wind dynamic pressure, and (d) the $\mathrm{AE}$ and (e) $D_{s t}$ indices characterizing the magnetospheric response. There were three distinct $D_{s t}$-enhancements during that period, one on 2 May at about 04:00 UT, the largest one on 4 May at about 04:00 UT, and the last one on 5 May at about 02:00 UT. The activity on 2 May was driven by a magnetic cloud, whose effects were first seen at about 03:35 UT. The IMF $B_{z}$ was close to zero but fluctuating until after 08:00 UT, then remained southward and at about $-10 \mathrm{nT}$ for more that $8 \mathrm{~h}$. The solar wind velocity was around $600 \mathrm{~km} / \mathrm{s}$. There were several pressure pulses reaching up to about $15 \mathrm{nPa}$. The strongest period of activ-

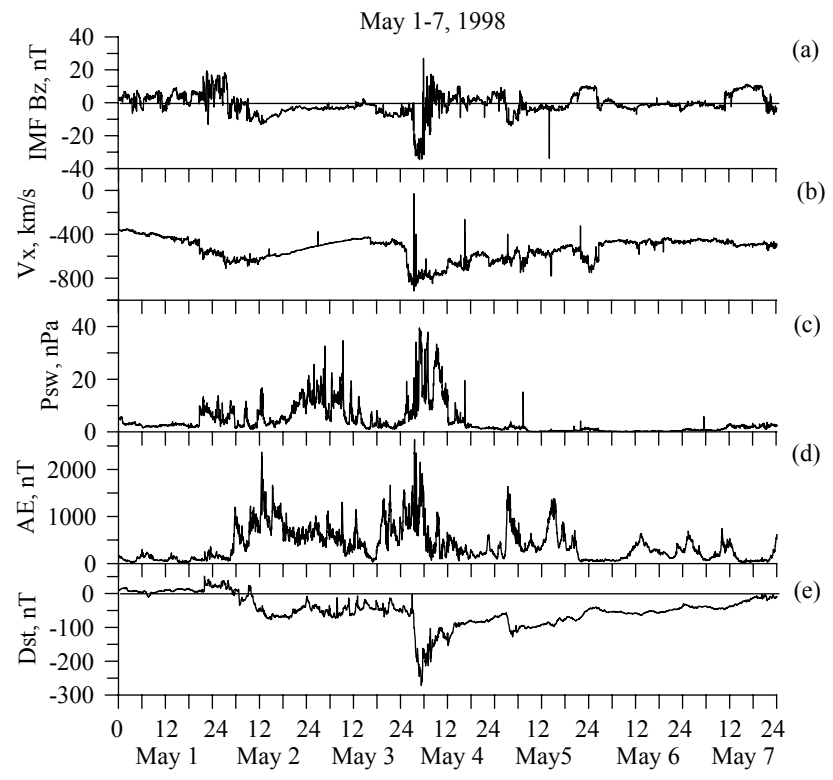

Fig. 3. 1-7 May 1998 storm event overview.

ity occurred on 4 May, when $B_{z}$ decreased sharply at about 02:30 UT down to $-30 \mathrm{nT}$, remained at that level for about three hours, and then increased and fluctuated around zero until the end of the day. The solar wind velocity increased to $800 \mathrm{~km} / \mathrm{s}$. The solar wind dynamic pressure was at its highest at about $40 \mathrm{nPa}$ around 04:00 UT and $30 \mathrm{nPa}$ around 08:00 UT. During the period between 04:00-08:00 UT the magnetopause crossed the geosynchronous orbit repeatedly, getting as close as $5 R_{E}$ in the subsolar region (Russell et al., 2000). On 5 May there was one more extended period of southward IMF at the beginning of the day, after that $B_{z}$ was mostly small or positive throughout the rest of the period. The magnetospheric response was seen as a strong increase in the $A_{E}$ index that reached over $2000 \mathrm{nT}$ at about 12:00 UT on 2 May and at about 04:00 UT on 4 May. The $D_{s t}$ index reached about $-80 \mathrm{nT}$ at 15:00 UT and recovered to the level of about $-50 \mathrm{nT}$ by the end of the day on 2 May. On 4 May, the $D_{s t}$ index decreased to $-250 \mathrm{nT}$, followed by a slow recovery toward a more quiet-time state.

\subsection{Energetic particle response}

Figure 4 shows (a) the measured $D_{s t}$ index, the ring current energies in Joule calculated for the protons for (b) all (1-200 keV), (c) low (1-20 keV), (d) medium (20-80 keV), and (e) high (80-200 keV) energies using the Polar CAMMICE/MICS measurements during the period of 1-7 May 1998 storm. The contributions to the total ring current energy from the protons at different energies behave in the same manner as obtained in the statistical study in the previous section. During the main phase of 2 May and 4 May storm activations, the main contribution comes from the medium energies (about $5 \cdot 10^{14} \mathrm{~J}$ from the medium energies and about $1 \cdot 10^{14} \mathrm{~J}$ from the high energies to the $8 \cdot 10^{14} \mathrm{~J}$ of all energies). 
The high energy protons contribute most during the recovery phase (about $1.5-3 \cdot 10^{14} \mathrm{~J}$ from high energies and about $0.6-1 \cdot 10^{14} \mathrm{~J}$ from medium energies to the $4-6 \cdot 10^{14} \mathrm{~J}$ of all energies).

\section{2-4 May 1998 storm event: modeling results}

\subsection{Particle tracing in the large-scale time-varying fields}

In order to model the contributions from protons at different energy ranges to the total ring current energy during the storm, we traced protons with $90^{\circ} \pm 60^{\circ}$ pitch angles under the conservation of the 1st and 2nd adiabatic invariants in different time-dependent magnetic and electric fields. We set an initial distribution function as an isotropic Maxwellian distribution function with the observed density and average temperature, at a boundary $R=8,19: 00-05: 00$ MLT in the equatorial plane. We compute the drift velocity as a combination of the velocity due to $\boldsymbol{E} \times \boldsymbol{B}$ drift, and the bounceaveraged velocity due to magnetic drift, including gradient and curvature drifts (Roederer, 1970)

$\left\langle v_{0}\right\rangle=\frac{\boldsymbol{E}_{\mathbf{0}} \times \boldsymbol{B}_{\mathbf{0}}}{B_{0}^{2}}+\frac{2 p}{q \tau_{b} B_{0}} \nabla I \times \mathbf{e}_{\mathbf{0}}$,

where

$I=\int_{S_{m}}^{S_{m}^{\prime}}\left[1-\frac{B(s)}{B_{m}}\right]^{1 / 2} d s$

where $\boldsymbol{E}_{\mathbf{0}}$ and $\boldsymbol{B}_{\mathbf{0}}$ are electric and magnetic fields in the equatorial plane, respectively, $p$ is the particle momentum, $q$ is the particle charge, $\tau_{b}$ is the bounce period, $\boldsymbol{e}_{\mathbf{0}}$ is the unit vector, $S_{m}$ and $S_{m}^{\prime}$ are the mirror points, $B(s)$ is the magnetic field along magnetic field line, $B_{m}$ is the magnetic field at the mirror point and $d s$ is the magnetic field line length element.

We obtain the distribution function at the next time moment, assuming the Liouville theorem about conservation of the distribution function along the dynamic trajectory of particles but taking into account the losses, such as chargeexchange with $\tau_{\text {loss }}=\frac{1}{\sigma n V}$. The charge-exchange cross section $\sigma$ is given by Janev and Smith (1993) and the number density $n$ of neutrals is given by the thermospheric model MSISE 90 (Hedin, 1991).

Figure 5 presents the calculated proton ring current energy in Joule for four energy ranges, total $(1-200 \mathrm{keV}$, black curves), low (1-20 keV, blue curves), medium (20-80 keV, green curves) and high energies (80-200 keV, red curves). The measured $D_{s t}$ index is shown in the bottom panel for the period of 2-4 May 1998.

Figure 5a shows results from tracing protons in the dipole magnetic field and the $K_{p}$-dependent Volland-Stern electric field. Constant plasma sheet number density $N_{p s}=0.4 \mathrm{~cm}^{-3}$ and average temperature $\left\langle T_{p s}>=5 \mathrm{keV}\right.$ were used for the initial distribution at $R=8,19: 00-05: 00$ MLT. The VollandStern (Volland, 1973; Stern, 1975) electric potential $\Phi_{\text {conv }}$ is given by

$\Phi_{\mathrm{conv}}=A L^{\gamma} \sin \left(\phi-\phi_{0}\right)$,

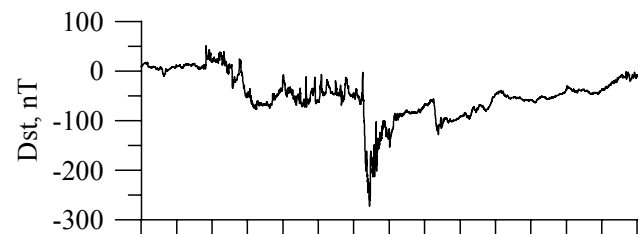

(a)

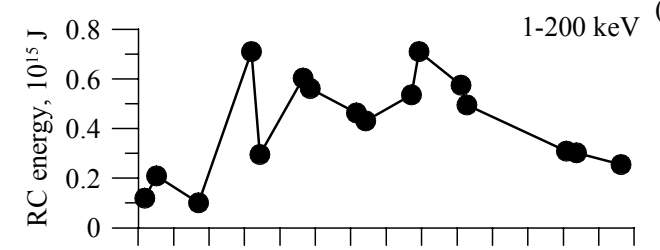

(b)
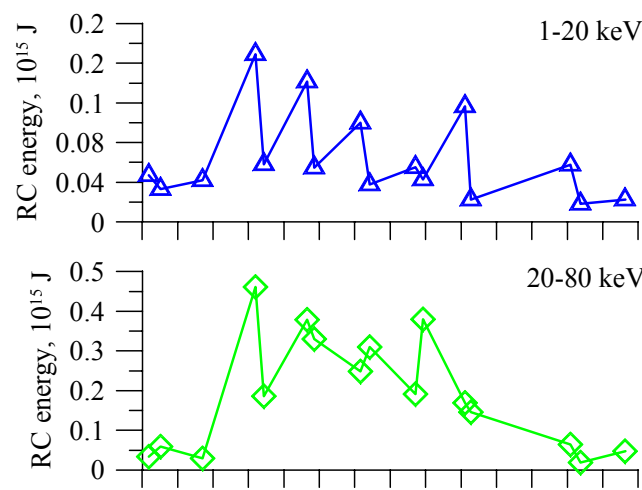

(d)

(c)

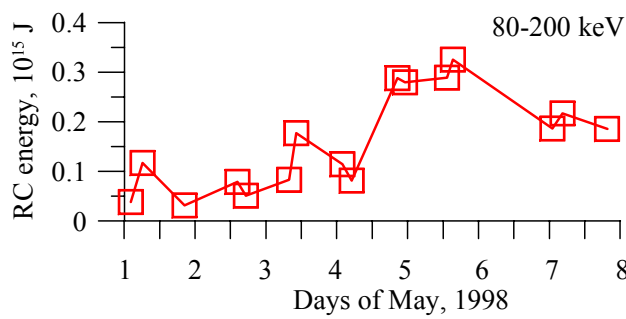

(e)

Fig. 4. Measured $D_{s t}$ index (a) and calculated proton ring current energies using Polar CAMMICE/MICS measurements from (b) all (1-200 keV), (c) low (1-20 keV), (d) medium (20-80 keV), and (e) high (80-200 keV) energy protons during the period of 1-7 May 1998.

where $A$ determines the intensity of the convection electric field, $\gamma$ is the shielding factor, $\phi$ is the magnetic local time, and $\phi_{0}$ is the offset angle from the dawn-dusk meridian. We used a $K_{p}$-dependent function for $A$ (Maynard and Chen, 1975)

$A=\frac{0.045}{\left(1-0.159 K_{p}+0.0093 K_{p}^{2}\right)^{3}} k V / R_{E}^{2}$,

where $\gamma=2$ and $\phi_{0}=0$. The main contribution to the total ring current energy comes from the protons with medium energies (20-80 keV) during the entire modelled storm period. The contribution from the high energies is very small. This implies that the $K_{p}$-dependent convection enhancement does not produce the observed acceleration to high energies during storm recovery phase.

The ring current energy curves shown in Fig. 5b were calculated by tracing protons under the same conditions as in 


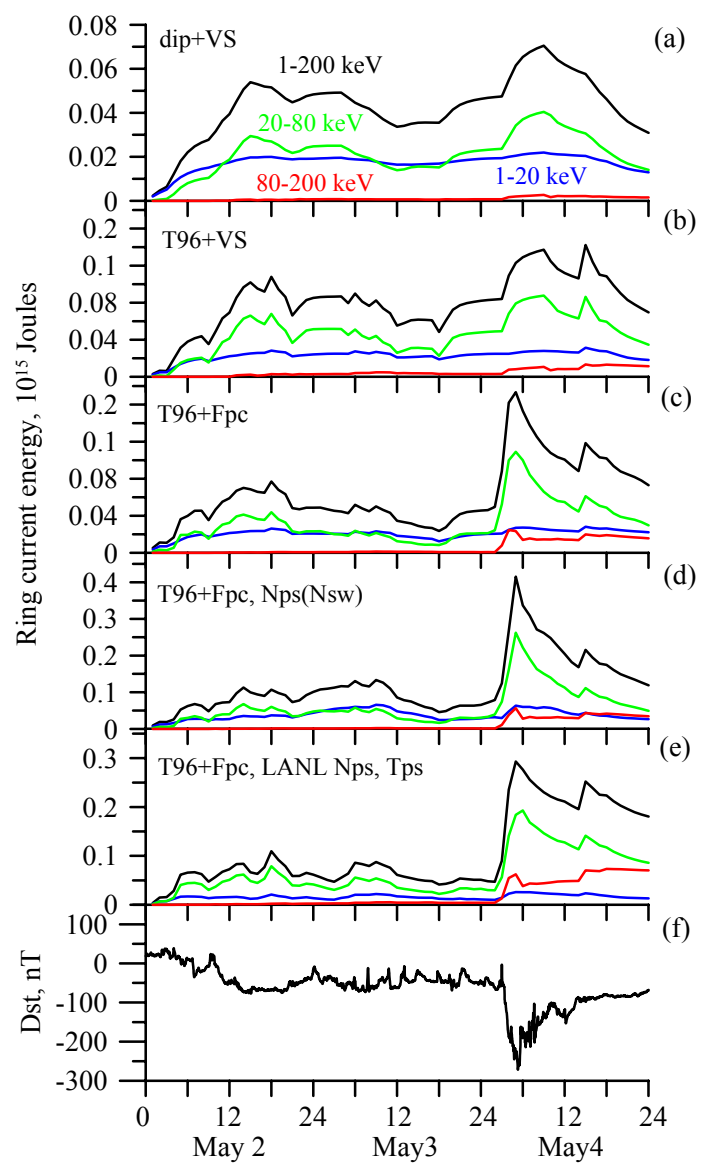

Fig. 5. Calculated proton ring current energy in Joule for total (1-200 keV, black curves), low (1-20 keV, blue curves), medium (20-80 keV, green curves) and high energies $(80-200 \mathrm{keV}$, red curves) while tracing in (a) dipole magnetic field and Volland-Stern electric field with constant plasma sheet number density and average temperature as initial conditions, (b) Tsyganenko T96 magnetic field instead of dipole, (c) Boyle et al. (1997) polar cap potential applied to Volland-Stern model instead of Volland-Stern electric field, (d) Tsyganenko T96 magnetic field and Boyle et al. (1997) polar cap potential applied to Volland-Stern model with the plasma sheet number density dependent on the solar wind number density and average temperature as initial conditions, and (e) with the plasma sheet number density and average temperature obtained from corresponding LANL particle measurements, together with the measured $D_{s t}$ index (f) for 2-4 May 1998.

Fig. 5a, but using a more realistic Tsyganenko T96 (Tsyganenko, 1995) magnetic field model instead of the pure dipole. The T96 model was used with the one-hour averaged input parameters $D_{s t}, P_{s w}$, IMF $B_{y}$ and $B_{z}$ observed by the WIND spacecraft. The time delay which corresponds to the travel time of the solar wind between WIND and magnetopause was taken into account. It can be seen that using a more realistic, non-dipole, varying magnetic field results in an almost two times increase of the total and medium ring current energies. This indicates the importance of using non-dipole magnetic field models in particle tracing. At the same time, the contribution from the high energy population is rather small.

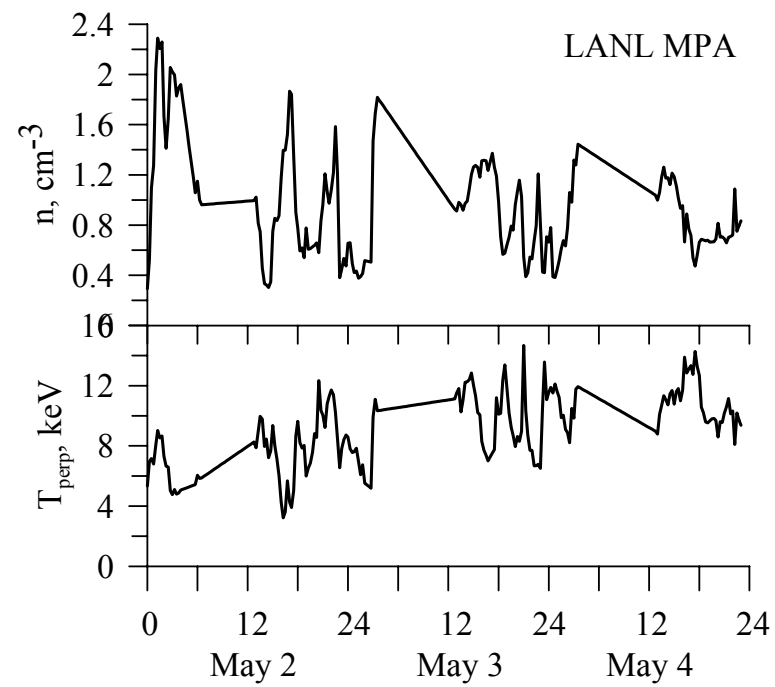

(a)

Fig. 6. (a) Plasma sheet number density and (b) averaged perpendicular temperature used as boundary conditions for the modeled period of 2-4 May 1998 obtained from the MPA instrument data on board the Los Alamos (LANL) geosynchronous orbit satellites.

Figure 5c shows the results of the proton tracing in the Tsyganenko T96 magnetic field and the Boyle et al. (1997) polar cap potential mapped to the magnetotail. Following Ebihara and Ejiri (2000), the Boyle et al. (1997) function for the polar cap potential $F$ coupled with the solar wind parameters and applied to the Volland-Stern type convection gives

$\Phi_{p c}=\left[1.1 \cdot 10^{-4} V_{s w}^{2}+11.1 \cdot B_{\mathrm{IMF}} \sin ^{3}\left(\frac{\theta_{\mathrm{IMF}}}{2}\right)\right] \frac{\sin \phi}{2}\left(\frac{R}{R_{B}}\right)^{2}$,

where $V_{s w}$ is the solar wind bulk velocity, $B_{\mathrm{IMF}}$ is the interplanetary magnetic field, $\theta_{\mathrm{IMF}}=\tan ^{-1}\left(B_{\mathrm{ZIMF}} / B_{\mathrm{yIMF}}\right)$ is the IMF clock angle, $R$ is the radial distance, and $R_{B}=10.47 R_{E}$. This model produces a sharp increase in the ring current energy during the 4 May storm, but the contribution from the high energies does not increase sufficiently to dominate during the storm recovery.

The influence of changing the initial conditions is shown in Fig. 5d, where we computed the plasma sheet number density from the solar wind number density as $N_{p s}=0.025 N_{s w}+0.395$ (Ebihara and Ejiri, 2000). The temperature was held constant at $5 \mathrm{keV}$. For producing of Fig. $5 \mathrm{e}$, we used the data from the MPA instrument (McComas et al., 1993) on board the Los Alamos (LANL) geosynchronous satellites to obtain the plasma sheet number density and average temperature for ions in the energy range $0.1-40 \mathrm{keV}$. The time series of the number density and averaged perpendicular temperature shown in Fig. 6 are created from measurements obtained within $4 \mathrm{~h}$ of local time around midnight. Values were averaged when more than one spacecraft was simultaneously in that region. When no satellites were near midnight, the data were interpolated linearly. These values were then used as time-dependent boundary conditions. It is evident from Figs. 5d and e that changing the initial conditions 


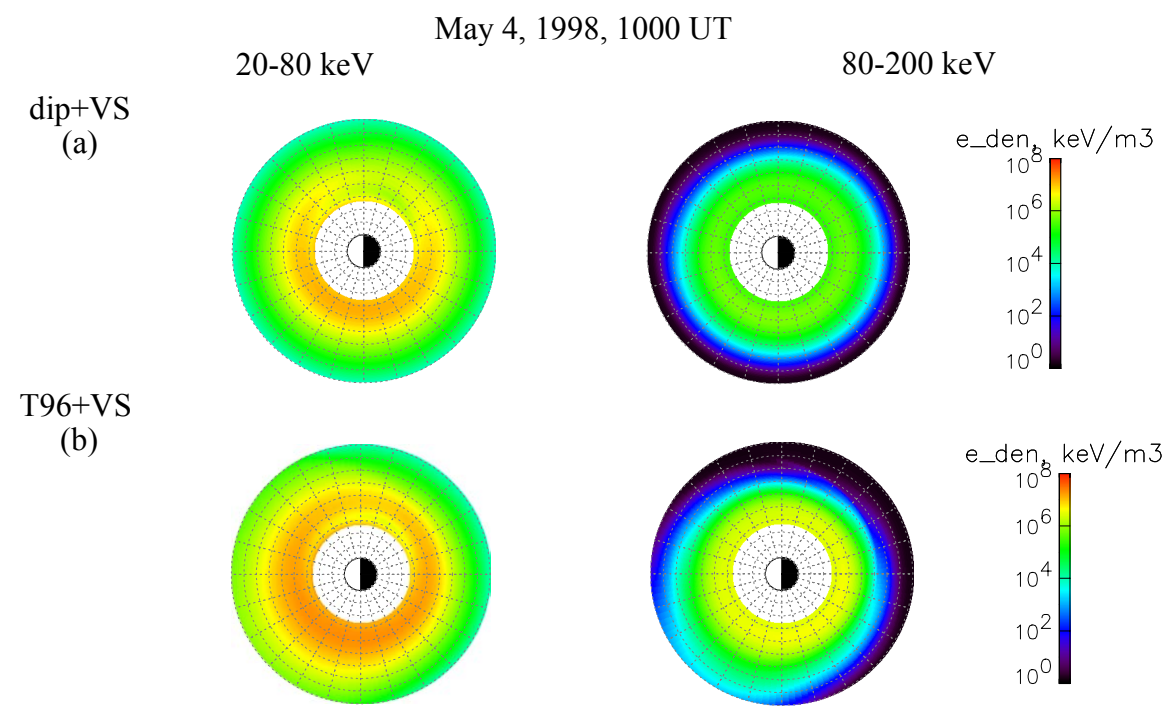

T96+Fpc

(c)
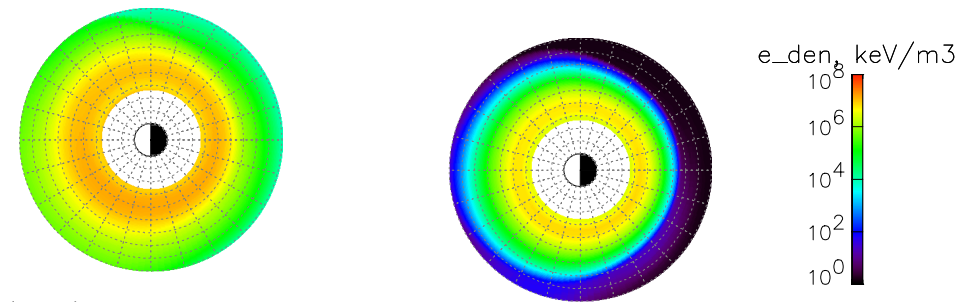

T96+Fpc, Nps(Nsw)

(d)
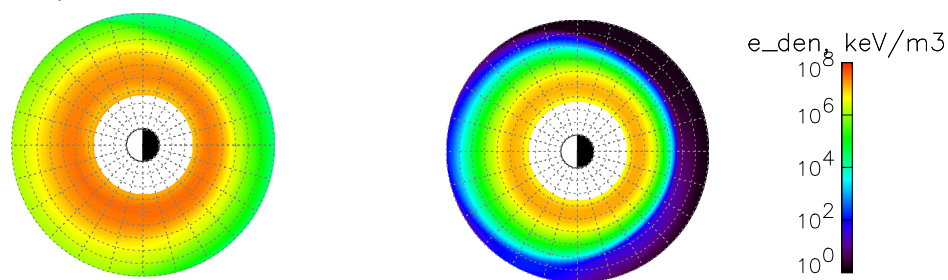

T96+Fpc, LANL Nps, Tps

(e)
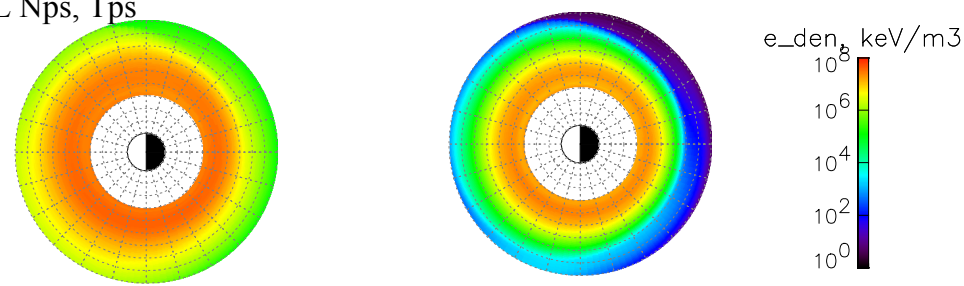

Fig. 7. Calculated energy density maps in the equatorial plane for protons with medium (20-80 keV) energies and high (80-200 keV) energies while tracing in 5 different combinations of field models and initial conditions similar to those shown in Figs. 5a-e during the recovery phase of 4 May 1998 storm at 10:00 UT.

does not provide dominant contribution from the high energies during the storm recovery.

Figure 7 shows the calculated energy density maps in the equatorial plane for protons with medium $(20-80 \mathrm{keV})$ energies and high (80-200 keV) energies for the five different combinations of field models and initial conditions shown in Figs. 5a-e. The time instant shown is 4 May 1998 at 10:00 UT, during the storm recovery phase. The intensity of the high energy ring current formed (right panels) is much less than that of the medium energies (left panels).
4.2 2-4 May 1998: effects of substorm-associated impulsive electric and magnetic fields

In order to further examine the particle energization, we introduced transient fields associated with the dipolarization process in the magnetotail during substorm onset. The dipolarization was modeled as an earthward propagating electromagnetic pulse of localized radial and longitudinal extent ( $\mathrm{Li}$ et al., 1998; Sarris et al., 2002). The electric field was given as a time-dependent Gaussian pulse with a purely azimuthal 
electric field component that decreases away from midnight. The earthward propagation speed decreased as the pulse moved inward to mimic the breaking of the flows (Shiokawa et al., 1997). In the spherical coordinate system $(r, \theta, \phi)$, where $r=0$ at the center of the Earth, $\theta=0$ defines the equatorial plane and $\phi=0$ is at local noon (positive eastward). The electric field is given by

$$
\mathbf{E}_{\phi}=-\hat{e}_{\phi} E_{0} / E_{\max }\left(1+c_{1} \cos \left(\phi-\phi_{0}\right)\right)^{p} \exp \left(-\xi^{2}\right),
$$

where $\xi=\left[r-r_{i}+v(r)\left(t-t_{a}\right)\right] / d$ determines the location of the maximum value of the pulse, $v(r)=a+b r$ is the pulse front velocity as a function of radial distance $r, d$ is the width of the pulse, $c_{1}(>0)$ and $p(>0)$ describe the local time dependence of the electric field amplitude, which is largest at $\phi_{0}, t_{a}=\left(c_{2} / v_{a}\right)\left(1-\cos \left(\phi-\phi_{0}\right)\right)$ represents the delay of the pulse from $\phi_{0}$ to other local times, $c_{2}$ determines the magnitude of the delay, $v_{a}$ is the longitudinal speed of the pulse (assumed constant), and $r_{i}$ is a parameter in the simulation that determines the arrival time of the pulse. We introduced a normalization coefficient $E_{\max }$ for the electric pulse amplitude, since using directly the Eq. (1) from Sarris et al. (2002) gives unrealistic numbers for maximum $E_{\phi}$ at midnight $\left(\phi=180^{\circ}\right)$, at $R=6.6 R_{E}$ $\left(E_{\max }(\exp =1)=4 \mathrm{mV} / \mathrm{m}(1+\cos (1))^{8}=1024 \mathrm{mV} / \mathrm{m}\right)$. Following Sarris et al. (2002), we used

$\phi_{0}=0, c_{1}=1, c_{2}=0.5 R_{E}, a=53.15 \mathrm{~km} / \mathrm{s}, b=0.0093 \mathrm{~s}^{-1}$,

$p=8, v_{a}=20 \mathrm{~km} / \mathrm{s}, r_{i}=100 R_{E}, d=4 \cdot 10^{7} \mathrm{~m}$.

The magnetic field disturbance from this dipolarization process was obtained from Faraday's law $(\partial \boldsymbol{B} / \partial t=-\nabla \times \boldsymbol{E})$. The total fields are always used in the drift velocity calculations.

We launched several pulses at the substorm onset times during the period of 2-4 May 1998. Assuming a baseline value $E_{0}=4 \mathrm{mV} / \mathrm{m}$ for an AE index of $1000 \mathrm{nT}$ (Sarris et al., 2002), we set the ratio of the pulse amplitudes similar to the ratios of the peak values in the $\mathrm{AE}$ index. Table 1 contains the times and magnitudes of $E_{0}$ of the launched pulses.

It is necessary to mention that after the pulse has gone, there exists a residual magnetic field, Fig. 1, Li et al. (1998). This is the magnetic field from the pulse which does not disappear but continues to contribute to the total magnetic field. When a set of pulses is launched, the residual magnetic field causes non-realistic gradients in the total magnetic field and non-realistic behavior of particle trajectories. Therefore, we have introduced a dumping mechanism that switches on a decay of the disturbance magnetic field from the pulse with a dumping decrement $\tau$. This moment corresponds to the end of the active phase of the pulse. It is determined from the ratio between the magnetic field changes with time $\frac{\partial \boldsymbol{B}}{\partial \boldsymbol{t}}$ (computed from the Maxwell equation $\frac{\partial \boldsymbol{B}}{\partial \boldsymbol{t}}=-\nabla \times \boldsymbol{E}$ ), and the magnetic field changes, which provide a decrease in the current intensity of the magnetic field $\mathrm{B}$ by a factor of $e$ during the time of $\tau=15 \mathrm{~min}$. If this ratio is smaller than 1 , then the magnetic field from the pulse starts to decrease as
$B(t)=B(t=0) \exp (-t / \tau)$. The physical interpretation of this procedure is that after becoming more dipole during the substorm onset, the magnetic field lines should return to their more tail-like configuration during the substorm recovery.

Figure 8 shows the calculated energy density maps in the equatorial plane for protons with medium $(20-80 \mathrm{keV})$ energies when the particle tracing was performed using the Tsyganenko T96 magnetic field model, the Boyle et al. (1997) polar cap potential applied to the Volland-Stern model, with the plasma sheet number density and averaged perpendicular temperature obtained from LANL MPA data (Fig. 6), and with addition of thirteen electromagnetic pulses at the observed substorm onset times during 2-4 May 1998. The time instant is marked by the red line on the $D_{s t}$ curves and is placed under each of the energy density maps. The empty magnetosphere starts to fill (Fig. 8a) and the effects of the incoming pulses can be seen (Figs. 8b,c). Medium energy protons formed a symmetric ring current at the end of 2 May (Fig. 8d). Stronger pulses acted during the main phase of the 4 May storm. Influence of multiple pulses can be noted in Fig. 8f as several particle populations appear detached from each other. During the recovery phase (Fig. 8h) the ring current with peak energy densities about $2 \cdot 10^{7} \mathrm{keV} / \mathrm{m}^{3}$ became quite symmetric. Note that the color scale is different from that in Fig. 7.

Figure 9, similar to Fig. 8, shows the calculated energy density maps in the equatorial plane for protons with high $(20-80 \mathrm{keV})$ energies. The filling of the empty magnetosphere (Fig. 9b) and the effects of the coming pulses (Figs. 9c,f) can also be seen. The energy densities in the high energy ring current formed and became symmetric during the recovery phase (Fig. 9h). The peak values are about 2 times higher than in the medium energy ring current (Fig. 8h).

Figure 10, similar to Fig. 5, shows the calculated proton ring current energy in Joule for the four energy ranges. It is clear that the action of the electromagnetic pulses led to highly efficient transport and energization of the protons into the inner magnetosphere, which then resulted in the dominant contribution of high energy protons to the proton ring current energy during the recovery phase.

\section{Conclusions and discussion}

We have studied the role of substorm-associated impulsive electric fields in the transport and energization of the ring current particles to the energies above $80 \mathrm{keV}$. Throughout a double magnetic storm on 2-4 May 1998, we followed the evolution of contributions to the total energy (1$200 \mathrm{keV}$ ) of the ring current from protons in three different energy ranges, low (1-20 keV), medium (20-80 keV) and high (80-200 keV) energies. Measurements from Polar CAMMICE/MICS instrument showed that the medium energy protons are the main contributors to the total ring current energy during the storm main phase. During the recovery phase the high energy protons play a dominant role. 
May 2-4, 1998, medium energies $(20-80 \mathrm{keV})$

(a)
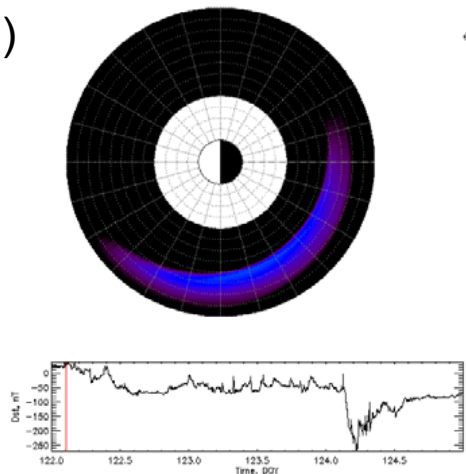

(c)
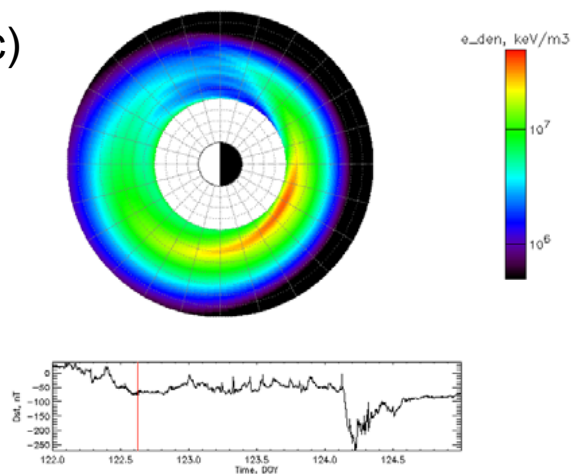

(e)
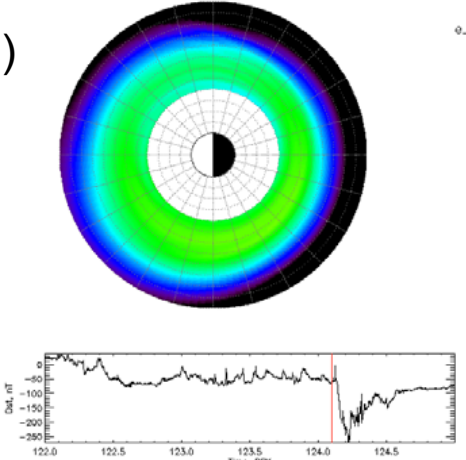

(g)
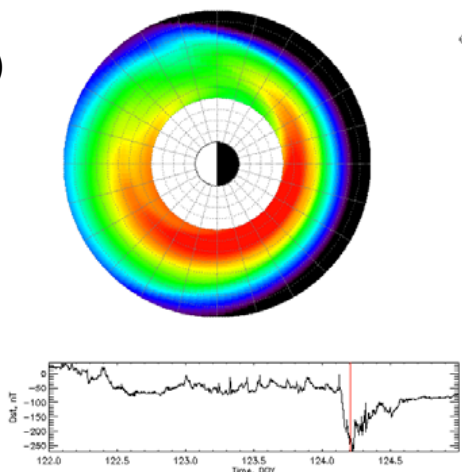

(b)
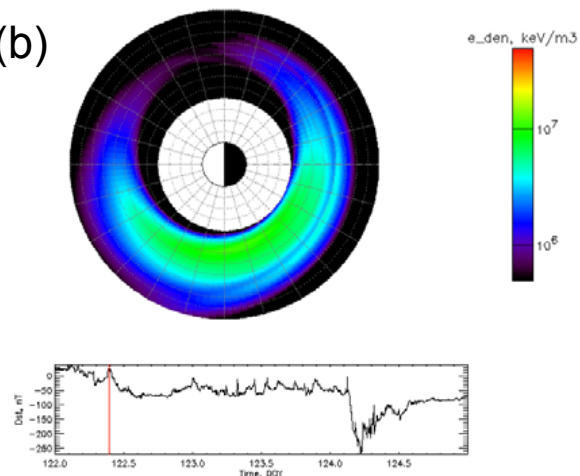

(d)
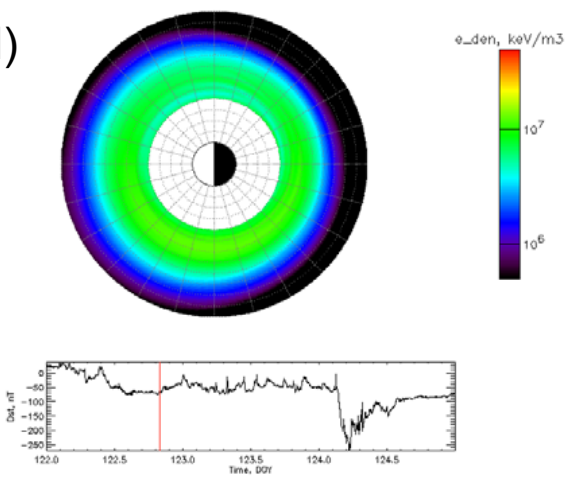

(f)
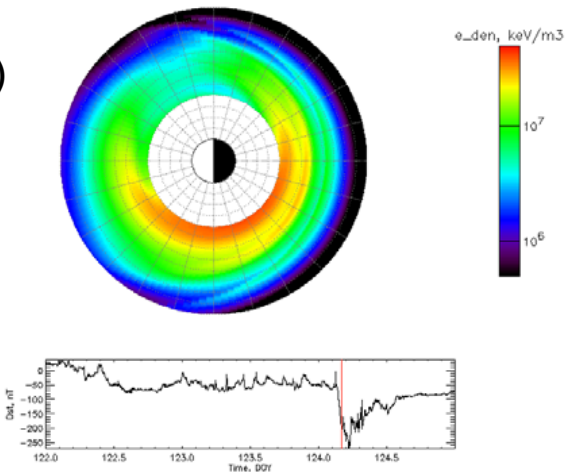

(h)
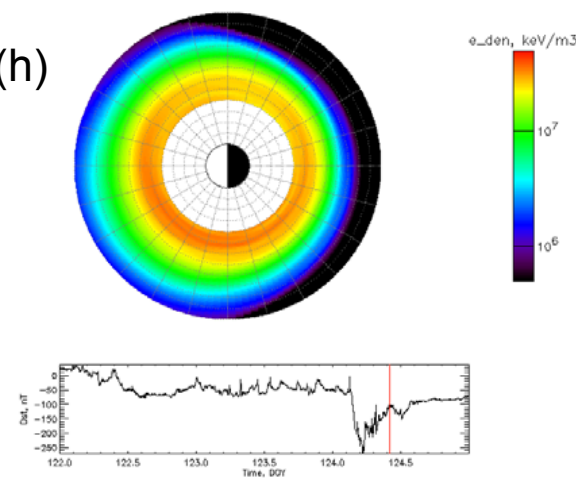

Fig. 8. Calculated energy density maps in the equatorial plane for protons with medium (20-80 keV) energies when several electromagnetic pulses were activated at the substorm onsets during the modelled period of 2-4 May 1998. 
May 2-4, 1998, high energies (80-200 keV)

(a)
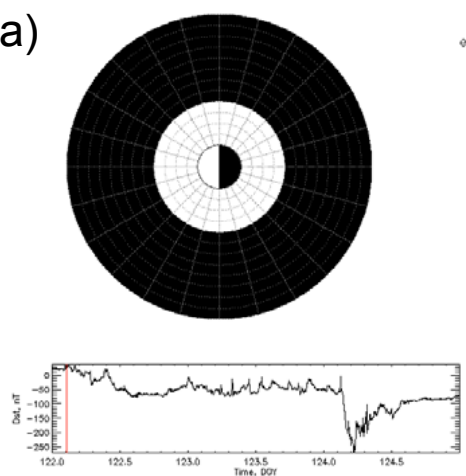

(c)
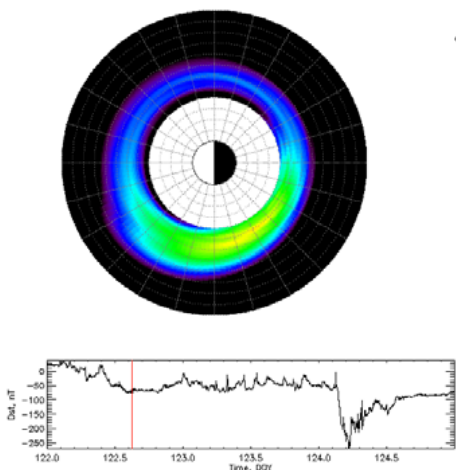

(e)
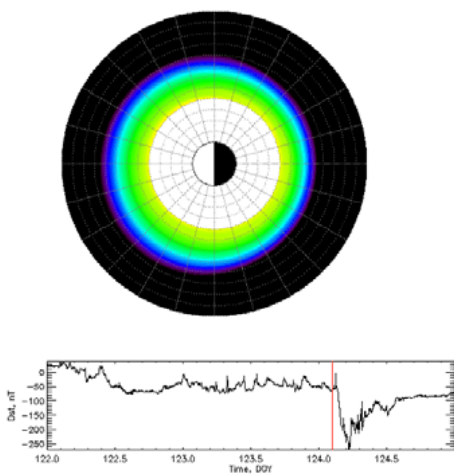

(g)
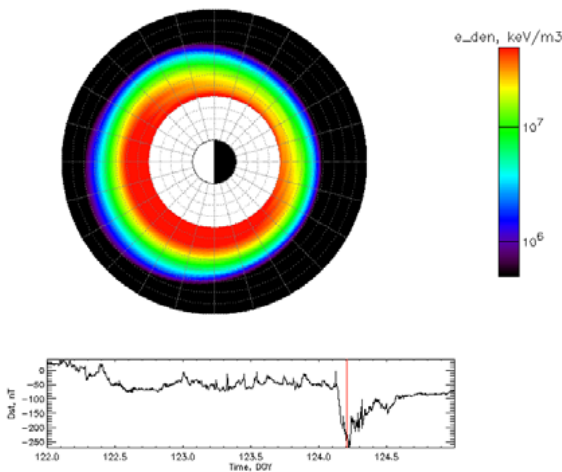

(b)
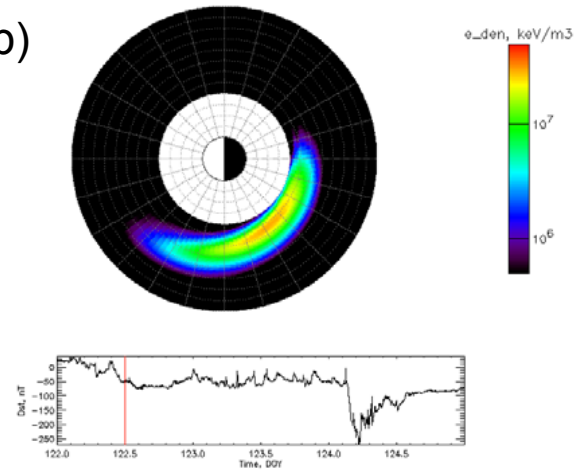

(d)
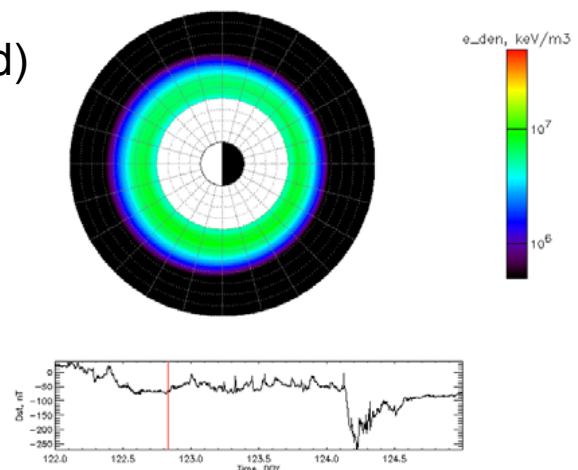

(f)
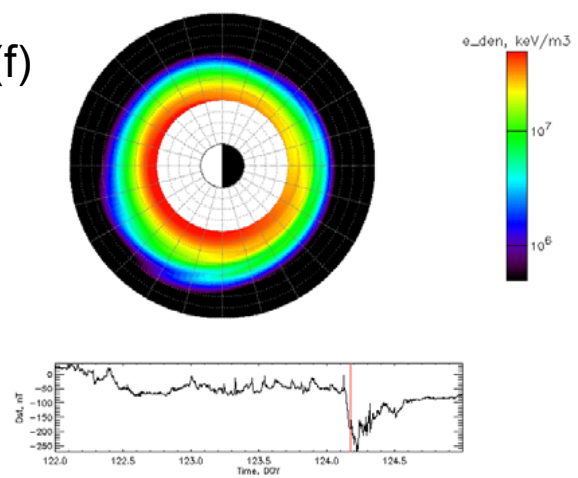

(h)
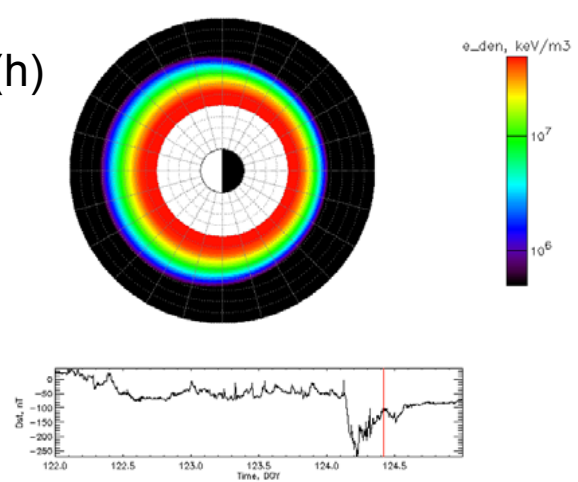

Fig. 9. Calculated energy density maps in the equatorial plane for protons with high ( $80-200 \mathrm{keV})$ energies when several electromagnetic pulses were activated at the substorm onsets during the modelled period of 2-4 May 1998. 
Table 1. Times and magnitudes of $E_{0}$ of the launched pulses at substorm onset times together with $\mathrm{AE}$ values during the period of 2-4 May 1998.

\begin{tabular}{lrc}
\hline UT & AE peak, nT & $E_{0}, \mathrm{mV} / \mathrm{m}$ \\
\hline 2 May 1998 & & \\
05:20 & 1000 & 4 \\
09:10 & 1000 & 4 \\
12:05 & 2000 & 8 \\
16:00 & 1500 & 6 \\
3 May 1998 & & \\
05:00 & 800 & 3 \\
12:00 & 800 & 3 \\
18:00 & 1000 & 4 \\
20:30 & 1500 & 6 \\
4 May 1998 & & \\
00:15 & 1500 & 6 \\
02:40 & 1500 & 6 \\
03:25 & 2000 & 8 \\
04:30 & 1800 & 7 \\
09:00 & 800 & 3 \\
\hline
\end{tabular}

Further development of the particle tracing procedure described in Ganushkina and Pulkkinen (2002) made it possible to trace protons with arbitrary pitch angles, assuming conservation of the first and second adiabatic invariants, in several different time-dependent magnetic and electric fields. It was shown that the dominant role of the high-energy ions during the storm recovery phase cannot be obtained by simply using variable intensity of the large-scale convection electric field or by changing the initial distribution density and/or temperature. Only the impulsive localized substorm-associated electric fields were strong enough to yield the observed fluxes of high-energy particles. These results indicate that the formation of the ring current is a combination of enhanced largescale convection and pulsed inward shift and consequent energization of the ring current particles.

The relative importance of the large-scale convection electric field and the substorm-associated impulsive electric fields in the energization and transport of ions into the ring current is still an open question. Many storms have been simulated based on the convection paradigm (Lee et al., 1983; Takahashi et al., 1990; Kozyra et al., 1998; Ebihara and Ejiri, 2000; Jordanova et al., 2001; Liemohn et al., 2001). In these calculations the magnetic field was in most cases a dipole, and the electric field was taken to be the Volland-Stern or other empirical convection electric field model. These authors have concluded that the ion transport into the ring current can be accomplished by the enhanced large-scale convection electric field, and that the role of the substormassociated electric field in developing the ring current is only to enhance the intensity of the convection electric field (Ebihara and Ejiri, 2003). However, in most cases where the total

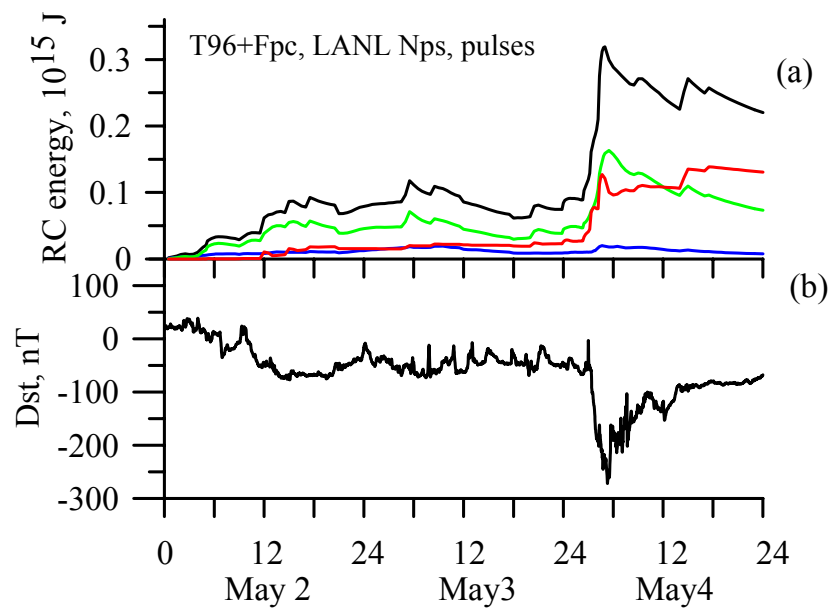

Fig. 10. Calculated proton ring current energy in Joule (a) for four energy ranges such as the total (1-200 keV, black curves), the low (1-20 keV, blue curves), the medium (20-80 keV, green curves) and high energies (80-200 keV, red curves) and the measured $D_{s t}$ index (b) for modelled period of 2-4 May 1998 when several electromagnetic pulses were activated at the substorm onsets.

energy density corresponds to that observed, the high-energy portion of the computed ring current is much weaker than the observations would indicate.

While the magnetospheric magnetic field is relatively well-known and several empirical models exist, the electric field observations are much fewer and the separation of the large-scale properties and the smaller scale impulsive structures is much less clear. Large-scale convection models describe the electric field either by associating the solar wind and IMF behavior with the ionospheric potential pattern and by mapping the ionospheric convection pattern to the tail (reconnection or viscous interaction as possible generators). Another possibility is to adjust the intensity of the convection field to the overall level of magnetic activity (plasma pressure gradients as possible generators). Both types of models yield large-scale stationary or a slowly varying tail electric field pattern. However, recent studies have shown that earthward transport of plasma and magnetic flux occurs in the form of short-duration, high-speed plasma flows, which are associated with magnetic field dipolarization and highly fluctuating electric fields, rather than as a slow, steady convection (Baumjohann et al., 1990; Angelopoulos et al., 1992; Sergeev et al., 1996). While these bursts occur during activity conditions, they become stronger and more numerous during periods of stronger activity.

To energize the plasma sheet particles to higher energies it is necessary to have fluctuating fields in the form of localized electromagnetic pulses. Both fluctuations and the localization of fields are important. However, there are a few observations of the large-scale convection electric field with large amplitudes. Rowland and Wygant (1998) and Wygant et al. (1998) showed measurements of the dawn-dusk component of the electric field in the inner magnetosphere ranging 
from 0.05 to $1.5 \mathrm{mV} / \mathrm{m}$ as $K_{p}$ increased from 0 to 9 . During one major storm with $D_{s t}$ at about $-300 \mathrm{nT}$ electric fields reaching $6 \mathrm{mV} / \mathrm{m}$ lasted for time periods of one hour or more. There are no statistics nor adequate models to support that measurement during one storm. Introducing such large convection electric fields for particle tracing would provide large amounts of high energy particles. However, if the magnitude of $6 \mathrm{mV} / \mathrm{m}$ is applied to the entire magnetotail (assumed to be $40 R_{E}$ ), as it is in the large-scale convection electric field, the polar cap potential drop will be $1500 \mathrm{kV}$. Often observed values of about $200 \mathrm{kV}$ give the reasonable magnitude of the convection electric field as $0.8 \mathrm{mV} / \mathrm{m}$. The introduction of such large electric fields is possible only in localized region.

Several studies have concluded that concurrent action of global convection and substorm-associated dipolarization and electric field variations inject plasma closer to the Earth than either one would do individually (Fok et al., 1999; Pulkkinen et al., 2000; Ganushkina and Pulkkinen, 2002). Ganushkina et al. $(2000,2001)$ studied the penetration of the plasma sheet ions into the inner magnetosphere using the Li et al. (1998) model, together with stationary electric and magnetic fields for particle tracing. They concluded that the inward displacement of these intense nose structures can occur under short-lived impulsive electric fields, combined with the convection electric field. On the other hand, large-scale convection alone leads to regular (lower energy) nose structures which take several (more than 5) hours to form if the magnetosphere remains sufficiently stationary (Buzulukova et al., 2003).

Earlier studies by Chapman (1962) and Akasofu (1966) regarded storms as superpositions of successive substorms. During the substorm expansion phase induction electric fields accelerate magnetospheric particles and inject them into the inner magnetosphere, where they become trapped and form the ring current. Later studies have stated that the substorm occurrence is incidental to the main phase of storms (Kamide et al., 1992). It is often assumed that storm-time substorms do not differ from non-storm substorms, but it has been noted that ions are energized more and penetrate deeper in the inner magnetosphere during storms than during isolated substorms (Daglis et al., 1998). However, a detailed study by Pulkkinen et al. (2002) showed that during magnetic storms, there are several distinct types of substorm-like activations, which are not similar to each other or to isolated substorms.

The present state of our understanding of the magnetospheric dynamics is quite advanced. Thus, gaining new understanding will necessarily require complex models of the electromagnetic fields and particle motion. This will require combination and coupling of multiple sources, as well as large-scale and small-scale processes. This study has clearly demonstrated the need to also include smaller-scale temporal variations in modeling of the large-scale evolution of the storm-time ring current.
Acknowledgements. We would like to thank K. Ogilvie and R. Lepping for the use of WIND data in this paper, World Data Center C2 for Geomagnetism, Kyoto, for the provisional $A_{E}, K_{p}$ and $D_{s t}$ indices data. The data were obtained from the Coordinated Data Analysis Web (CDAWeb). LANL MPA data was provided and used by permission by M. Thomsen. This work was supported by Academy of Finland. We are also very thankful to M. Ejiri and Y. Ebihara for the most useful discussions during two visits by N. Ganushkina of National Institute of Polar Research, Tokyo, Japan.

Topical Editor in chief thanks V. Sergeev and another referee for their help in evaluating this paper.

\section{References}

Aggson, T. L., Heppner, J., and Maynard, N.: Observations of large magnetospheric electric fields during the onset phase of a substorm, J. Geophys. Res., 88, 3981-3990, 1983.

Akasofu, S.-I.: The development of geomagnetic and auroral storms, J. Geomag. Geoelect., 18, 109-123, 1966.

Angelopoulos, V., Baumjohann, W., and Kennel, C. F. et al.: Bursty bulk flows in the inner central plasma sheet, J. Geophys. Res., 97, 4027-4039, 1992.

Arnoldy, R. L. and Chan, K. W.: Particle substorms observed at the geostationary orbit, J. Geophys. Res., 74, 5019-5028, 1969.

Baker, D. N., Higbie, P. R., Fritz, T. A. et al.: Observations and modeling of energetic particles at synchronous orbit on 29 July 1977, J. Geophys. Res., 87, 5917-5932, 1982.

Baumjohann, W., Paschmann, G., and Luehr, H.: Characteristics of high-speed ion flows in the plasma sheet, J. Geophys. Res., 95, 3801-3809, 1990.

Birn, J., Thomsen, M. F., and Borovsky, J. E. et al.: Substorm ion injections: Geosynchronous observations and test particle orbits in three-dimensional dynamic MHD fields, J. Geophys. Res., 102, 2325-2341, 1997.

Boyle, C. B., Reiff, P. H., and Hairston, M. R.: Empirical polar cap potentials, J. Geophys. Res., 102, 111-125, 1997.

Buzulukova, N. Y., Kovrazhkin, R. A., and Glazunov, A. L. et al.: Stationary nose structures of protons in the inner magnetosphere: Observations by the ION instrument on board the Interball-2 satellite and modeling, Cosmic Research, 41, 3-12, 2003.

Cattell, C. A. and Mozer, F. S.: Substorm electric fields in the Earth's magnetotail, in Magnetic Reconnection in Space and Laboratory Plasmas, Geophys. Monogr. Ser., vol. 30, edited by Hones, E. W. Jr., AGU, Washington, D.C., 208-215, 1984.

Chapman, S.: Earth Storms: Retrospect and Prospect, J. Phys. Soc. Japan, 17, 6-16, 1962.

Daglis, I. A., Kamide, Y., Kasotakis, G., Mouikis, C., Wilken, B., Sarris, T., and Nakamura, R.: Ion composition in the inner magnetosphere: Its importance and its potential role as a discriminator between storm-time substorms and non-storm substorms, in: Fourth International Conference on Substorms (ICS-4), edited by S. Kokubun and Y. Kamide, Terra/Kluwer Publications, Tokyo, 767-772, 1998.

Ebihara, Y. and Ejiri, M.: Simulation study on fundamental properties of the storm-time ring current, J. Geophys. Res., 105, 15 843-15 860, 2000.

Ebihara, Y. and Ejiri, M.: Numerical simulation of the ring current: Review, Space Sci. Rev., 105, 377-452, 2003.

Fok, M.-C., Moore, T. E., and Delcourt, D. C.: Modeling of inner plasma sheet and ring current during substorms, J. Geophys. Res., 104, 14 557-14 569, 1999. 
Ganushkina, N. Yu. and Pulkkinen, T. I.: Particle tracing in the inner Earth's magnetosphere and the formation of the ring current during storm times, Adv. Space Res., 30, 1817-1820, 2002.

Ganushkina, N. Yu., Pulkkinen, T. I., and Sergeev, V. A. et al.: Entry of plasma sheet particles into the inner magnetosphere as observed by Polar/CAMMICE, J. Geophys. Res., 105, 25205 $25219,2000$.

Ganushkina, N. Yu., Pulkkinen, T. I., Bashkirov, V. F., Baker, D. N., and Li, X.: Formation of intense nose structures, Geophys. Res. Lett., 28, 491-494, 2001.

Hedin, A. E.: Extension of the MSIS thermosphere model into the middle and lower atmosphere, J. Geophys. Res., 96, 1159, 1991.

Janev, R. K. and Smith, J. J.: Cross sections for collision processes of hydrogen atoms with electrons, protons, and multiply-charged ions, in: Atomic and Plasma- Material Interaction Data for Fusion, Int. At. Energ. Agency, 4, 78-79, 1993.

Jordanova, V. K., Kistler, L. M., Farrugia, C. J., and Torbert, R. B.: Effects of inner magnetospheric convection on ring current dynamics: 10-12 March 1998, J. Geophys. Res., 106, 29705 29720, 2001.

Kamide, Y.: Is substorm occurrence a necessary condition for a magnetic storm?, J. Geomagn. Geoelectr., 44, 109-117, 1992.

Kozyra, J. U., Jordanova, V. K., and Borovsky, J. E. et al.: Effects of a high-density plasma sheet on ring current development during the 2-6 November 1993, J. Geophys. Res., 103, 26 285-26305, 1998.

Lee, L. C., Corrick, G., and Akasofu, S.-I.: On the ring current energy injection rate, Planet. Space Sci., 31, 901-911, 1983.

Li, X., Baker, D. N., and Temerin, M. et al.: Simulation of dispersionless injections and drift echoes of energetic electrons associated with substorms, Geophys. Res. Lett., 25, 3763-3766, 1998.

Liemohn, M. W., Kozyra, J. U., and Thomsen, M. F. et al.: Dominant role of the asymmetric ring current in producing the stormtime $D_{s t}$, J. Geophys. Res., 106, 10 883-10 904, 2001.

Maynard, N. C., Burke, W. J., Basinska, E. M. et al.: Dynamics of the inner magnetosphere near times of substorm onsets, J. Geophys. Res., 101, 7705-7736, 1996.

Maynard, N. C. and Chen, A. J.: Isolated cold plasma regions: Observations and their relation to possible production mechanisms, J. Geophys. Res., 80, 1009-1013, 1975.

McComas, D. J., Bame, S. J., and Barraclough, B. L. et al.: Magnetospheric plasma analyser: initial three-spacecraft observations from geosynchronous orbit, J. Geophys. Res., 98, 13 453-13 465, 1993.

McIlwain, C. E.: Substorm injection boundaries, in Magnetospheric Physics, edited by McCormac, B. M., Reidel, D., Norwell, Mass., 143, 1974.

Moore, T. E., Arnoldy, R. L., Feynman, J., and Hardy, D. A.: Propagating substorm injection fronts, J. Geophys. Res., 86, 67136726, 1981.

Pulkkinen, T. I., Ganushkina, N. Y., and Bashkirov, V. F. et al.: Ring current enhancement due to substorm-associated inductive electric fields, Proc. 5th Int. Conf. Substorms, ESA Publications Division, Special Publication SP-443, 451-454, 2000.
Pulkkinen, T. I., Ganushkina, N. Y., Baker, D. N., Turner, N. E., Fennell, J. F., Roeder, J., Fritz, T. A., Grande, M., Kellett, B., and Kettmann, G.: Ring current ion composition during solar minimum and rising solar activity: Polar/CAMMICE/MICS results, J. Geophys. Res., 106, 19 131-19 147, 2001.

Pulkkinen, T. I., Koskinen, H. E. J., and Huttunen, K. E. J. et al.: Effects of magnetic storms on substorm evolution, Proc. 6th Int. Conf. Substorms, edited by Winglee, R. M., 464-471, 2002.

Reeves, G. D., Belian, R. D., and Fritz, T.: Numerical tracing of energetic particle drifts, J. Geophys. Res., 96, 13 997-14 008, 1991.

Roederer, J. G.: Dynamics of geomagnetically trapped radiation, Springer-Verlag, New York, 36, 1970.

Rowland, D. E. and Wygant J. R.: Dependence of the large-scale, inner magnetospheric electric field on geomagnetic activity, J. Geophys. Res., 103, 14 959-14 964, 1998.

Russell, C. T., Le, G., and Chi, P. et al.: The extreme compression of the magnetosphere on 4 May 1998, as observed by the Polar spacecraft, Adv. Space Res., 25, 1369-1375, 2000.

Sarris, T. E, Li, X., Tsaggas, N., and Paschalidis, N.: Modeling energetic particle injections in dynamic pulse fields with varying propagation speeds, J. Geophys. Res., 107, CiteID 1033, DOI 10.1029/2001JA900166, 2002.

Sergeev, V. A., Angelopoulos, V., Gosling, J. T., Cattell, C. A., and Russell, C. T.: Detection of localized, plasma-depleted flux tubes or bubbles in the midtail plasma sheet, J. Geophys. Res., 101, 10 817-10 826, 1996.

Shiokawa, K., Baumjohann, W., and Haerendel, G.: Braking of high-speed flows in the near-Earth tail, Geophys. Res. Lett., 24, 1179-1182, 1997.

Stern, D. P.: The motion of a proton in the equatorial magnetosphere, J. Geophys. Res., 80, 595-599, 1975.

Takahashi, S., Iyemori, T., and Takeda, M.: A simulation of the storm-time ringcurrent, Planet. Space Sci., 38, 1133-1141, 1990.

Tsyganenko, N. A.: Modeling the Earth's magnetospheric magnetic field confined within a realistic magnetopause, J. Geophys. Res., 100, 5599-5612, 1995.

Tu, J.-N., Tsuruda, K., Hayakawa, H., Matsuoka, A., Mukai, T., Nagano, I., and Yagitani, S.: Statistical nature of impulsive electric fields associated with fast ion flow in the near-Earth plasma sheet, J. Geophys. Res., 105, 18 901-18 907, 2000.

Volland, H.: A semi-empirical model of large-scale magnetospheric electric field, J. Geophys. Res., 78, 171, 1973.

Wilken, B., Weiss, W., Hall, D., Grande, M., Soraas, F., and Fennell, J. F.: Magnetospheric ion composition spectrometer on board the CRRES spacecraft, J. Spacecr. Rockets, 29, 585-591, 1992.

Wygant, J., Rowland, D., Singer, H. J., Temerin, M., Mozer, F., and Hudson, M. K.: Experimental evidence on the role of the large spatial electric field in creating the ring current, J. Geophys. Res., 103, 29 527-29544, 1998.

Zaharia, S., Cheng, C. Z., and Johnson, J. R.: Particle transport and energization associated with substorms, J. Geophys. Res., 105, 18741-18 752, 2000. 